\title{
Synthesis of Polyphosphate Polymer Employing the Bisphenol (BHBF) and the Dichloride of Phenylphosphonic (PPDC): Evaluation of the Thermal Characteristics
}

\author{
Lucas Dominguini ${ }^{1 *}$, Karina Martinello², Michael Peterson ${ }^{3}$, Humberto Gracher Riella ${ }^{1}$ and Márcio \\ Antônio Fiori ${ }^{4}$
}

${ }^{1}$ Post-Graduation Program in Chemical Engineering, Universidade Federal de Santa Catarina (UFSC), Florianópolis, Brazil ${ }^{2}$ Department of Chemical Engineering, Universidade do Extremo Sul Catarinense (UNESC), Criciúma, Brazil

${ }^{3}$ Post-Graduation Program in Science and Materials Engineering, Universidade do Extremo Sul Catarinense (UNESC), Criciúma, Brazil

${ }^{4}$ Post-Graduation Program in Environmental Science and Post-Graduation Program in Technology and Management of the Innovation, Universidade Comunitária da Região de Chapecó (UNOCHAPECÓ), Chapecó, Brazil

\begin{abstract}
This work presents studies about the synthesis and thermal characterization of a new polymer synthetized from the chloride phenylphosphonic molecule containing. The interfacial polycondensation method was utilized for the synthesis at low temperature. As monomer were used the phenylphosphonic dichloride (PPDC) and the 4,4'-dihydroxybenzophenone (DHBP). The synthesis reactions were conducted at room temperature with heterogeneous medium and constant stirring. The polymer was characterized by FTIR, NMR ${ }^{31} \mathrm{P}$ and MALDI-TOF, which proved the formation of a compound polyphosphonate. DSC and TGA showed that the vitreous transition occurs at temperature of $66.0^{\circ} \mathrm{C}$ and that the value of $220.0^{\circ} \mathrm{C}$ is the degradation temperature of the polymer. After thermal degradation process the percentage of residual material is around $22.0 \mathrm{wt} \%$. These results suggest the possibility of application of this material as a flame retardant in polymer materials.
\end{abstract}

Keywords

Synthesis of polyphosphonated polymer, Polyphosphate polymer, Flame-retardants polymers, Thermal properties of polyphosphate polymer and Flame retardant compound

\section{Introduction}

Polymeric materials are used in many industrial segments. These materials are easily burned and release toxic compounds during the combustion reactions. Scientific communities have manifested high interest in this area. Due the broad application of these materials in many products, several studies have been realized with polymeric materials containing compounds with flame retardant properties [1-5]. The flame retardant compounds are studied and applied in the polymeric materials to delay its combustions reactions. Normally, these materials are referred as flame retardant additives [1].

Some halogenated molecules have the flame retardant property. These materials are incorporated into polymeric materials for retard the spread of flame. But, the products from these reactions combustion are toxic gases and cause damages to health. Thus, an efficient alternative is the application of compounds containing phosphorus in structures. The chemical groups containing phosphorus can have the flame retardant property and to present low toxicity $[2,3,6]$.

Molecules containing red phosphorus in its structure, inorganic phosphate and organophosphorus generally are good flames retardants. Red phosphorus structures have higher concentration of phosphorus elements, so it can be used in minor amounts compared to the others. However,

*Corresponding author: Lucas Dominguini, Post-Graduation Program in Chemical Engineering, Universidade Federal de Santa Catarina (UFSC), Florianópolis, SC - 88040-900, Brazil

Accepted: July 10, 2019

Published online: July 12, 2019

Citation: Dominguini L, Martinello K, Peterson M, et al. (2019) Synthesis of Polyphosphate Polymer Employing the Bisphenol (BHBF) and the Dichloride of Phenylphosphonic (PPDC): Evaluation of the Thermal Characteristics. Current Trends Anal Bioanal Chem 3(1):114-124 
Citation: Dominguini L, Martinello K, Peterson M, et al. (2019) Synthesis of Polyphosphate Polymer Employing the Bisphenol (BHBF) and the Dichloride of Phenylphosphonic (PPDC): Evaluation of the Thermal Characteristics. Current Trends Anal Bioanal Chem 3(1):114-124

present great disadvantage because during the combustion reaction releases the gas phosphine $\left(\mathrm{PH}_{3}\right)$ that is highly toxic [7].

Polyphosphates compounds are a special class of the inorganic phosphates. Its performance as flame retardant is based in an effective mechanisms of release of hydroxyl radicals of the type $\mathrm{HO} \bullet$ during the combustions reactions. This dehydration reaction favors the formation of a protective layer on the polymeric molecules and acts as a protection barrier to combustion reactions $[8,9]$.

Some works have been realized to synthesize polymers or oligomers containing phosphorus into its structure for these applications. The insertion of flame retardants elements directly in the polymeric chain has been a good method and the results has been good. The phosphorus elements impregnated in the polymer chain inhibits the ignition process and favors the formation of a protective layer between the polymer chain and the oxygen molecules [4]. Also, processes of combustions involving these polymers generates gases with low toxicity when compared with the polymers containing flame retardants additives based in halogens elements [2].

One way of obtaining a phosphorous polymer is by polycondensation synthesis methods that at low temperature the polymeric chains have a good linearity [5]. This is a condensation reaction where two molecules react and generate a complex polymeric molecule and water, for example [10]. Studies show that the flame retardant ability of these polymers depends of the amount of phosphorus groups present in the polymeric chain $[5,11,12]$.

In this context, the present work has the purpose describing the synthesis and characterization of a new phosphorated polymer obtained by phenylphosphonic compound as monomers. The synthetized molecules needs subsequently have detailed studies about their properties as flame retardants, but shows important and interesting results that indicates possible flame retardant ability.

\section{Experimental}

For the synthesis of the polyphenyl phosphonate containing 4,4'-dihydroxy-benzophenone(DHBP-polyphenyl phosphonate) were utilized the chemical reagents showed on the Table 1.
In first step of the synthesis was prepared a solution of $\mathrm{NaOH}$ with $0.70 \mathrm{M}$ in deionized water. In this solution was added a solution of Benzyltriphenyl-phosphonium chloride (BTPPC) with $0.44 \mathrm{M}$ solubilized in Dichloromethane (DCM). The resultant solution was agitated for 40 minutes at room temperature.

In the second steps was added a solution of 4,4'-dihydroxy-benzophenone (DHBP) $0.46 \mathrm{M}$, solubilized in DCM, and $1.40 \mathrm{~g}$ of Phenylphosphonic dichloride (PPDC). The new emulsion was agitated in a jacketed reactor during one hour, at temperature of $24.0^{\circ} \mathrm{C}$.

After these procedures the DHBP-polyphenyl phosphonate was precipitated with hexane. The precipitated compound was dried and purified by solvent evaporation in a vacuum oven during $24 \mathrm{~h}$. All procedures were realized at temperature of $24.0^{\circ} \mathrm{C}$.

DHBP-polyphenyl phosphonate was characterized by Fourier transform infrared spectroscopy (FTIR), Nuclear Magnetic Resonance for the Phosphorus (NMR 31P) and by Matrix-assisted laser desorption/Ionization spectroscopy (MALDI), Differential scanning calorimetric (DSC) and Thermo gravimetric analysis (TGA).

FTIR analyses were realized with a Spectrophotometer Shimadzu (IR Prestige-21) with the transmittance mode and the samples were prepared in circular tablets of $\mathrm{KBr}$. The RMN 31P of solid states was realized with a Spectrometer Bruker (AC200), with the samples solubilized in protonated DMSO and the MALDI-TOF MS spectra were obtained with an AB Sciex4800plus MALDI-TOF/TOF MS apparatus, equipped with Nd:YAG Laser (operated at $100 \mathrm{~Hz}$ ). The spectra were recorded in the reflection positive ion mode and externally calibrated with "TOF/TOF calibration" standard solution. The acceleration voltage was of $100 \mathrm{mV}$ and the spectra of 400 shots were averaged to improve the signal-to-noise ratio.

TGA analyses were realized with a Systems Shimadzu (TGA-50), at temperature range 25.0 to $900.0{ }^{\circ} \mathrm{C}$ and with heating rate of $10.0^{\circ} \mathrm{C} / \mathrm{min}$ in nitrogenous atmosphere. The thermograms were obtained in a Differential Scanning Calorimeter Perkin Elmer (Jade DSC). For the DSC measurements the samples were prepared with a polymeric mass of $5.0 \mathrm{mg}$ and the temperature range studied were of -20.0 to $230.0^{\circ} \mathrm{C}$. The heating/cooling rate was a $10^{\circ} \mathrm{C} . \mathrm{min}^{-1}$ in nitrogenous at-

Table 1: Chemical reagents utilized for the synthesis reactions of the DHBP-Polyphosphonate polymer.

\begin{tabular}{|l|l|l|l|l|l|l|}
\hline Reagent & Chemical Formula & Physical Aspects & Purity (\%) & Molar Mass (g) & Furnisher \\
\hline Phenylphosphonic dichloride & $\mathrm{C}_{6} \mathrm{H}_{5} \mathrm{Cl}_{2} \mathrm{OP}$ & PPDC & Liquid & 90.0 & 194.9 & Sigma Aldrich \\
\hline 4,4'-dihydroxy-benzophenone & $\left(\mathrm{HOC}_{6} \mathrm{H}_{4}\right)_{2} \mathrm{CO}$ & DHBP & Powder & 99.0 & 214.0 & Sigma Aldrich \\
\hline Benzyltriphenyl-phosphonium chloride & $\mathrm{C}_{25} \mathrm{H}_{22} \mathrm{CIP}$ & BTPPC & Powder & 99.0 & 491.9 & Sigma Aldrich \\
\hline 2,6-Dimethylphenol & $\mathrm{C}_{8} \mathrm{H}_{10} \mathrm{O}$ & DMF & Crystalized & 99.0 & 122.0 & Sigma Aldrich \\
\hline Hexane & $\mathrm{C}_{6} \mathrm{H}_{14}$ & $\mathrm{C}_{6} \mathrm{H}_{14}$ & Liquid & 99.0 & 86.18 & Lafan \\
\hline Sodium Hydroxide & $\mathrm{NaOH}^{\prime}$ & $\mathrm{NaOH}$ & Micropearl & 99.0 & 86.18 & Lafan \\
\hline Dichloromethane & $\mathrm{CH}_{2} \mathrm{Cl}_{2}$ & DCM & Liquid & 99.5 & 84.93 & F.MAIA \\
\hline
\end{tabular}


Citation: Dominguini L, Martinello K, Peterson M, et al. (2019) Synthesis of Polyphosphate Polymer Employing the Bisphenol (BHBF) and the Dichloride of Phenylphosphonic (PPDC): Evaluation of the Thermal Characteristics. Current Trends Anal Bioanal Chem 3(1):114-124

mosphere.

\section{Results and Discussion}

Figure 1 shows the global reactions proposed for the polymerizations of the DHBP-Polyphosphonate polymer. The reaction occurs by interactions between 4,4'-dihydroxybenzophenone (DHBP) and a Phenylphosphonic dichloride (PPDC) molecule, at room temperature $\left(25^{\circ} \mathrm{C}\right)$ for two hours under constant agitation.<smiles></smiles>

Figure 1: Global reaction proposed for the polymerization of the DHBP-Polyphosponate.

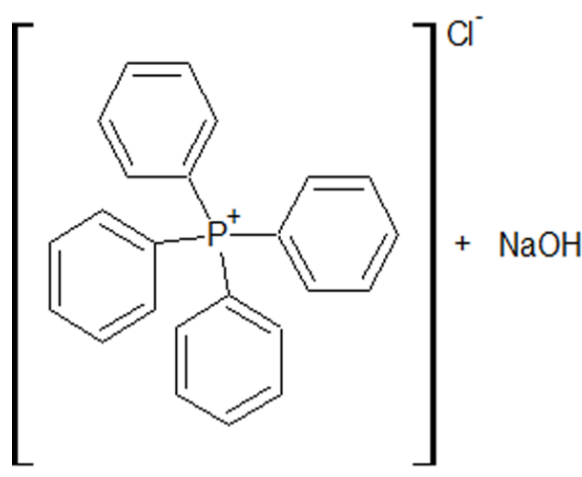

(BTPPC)

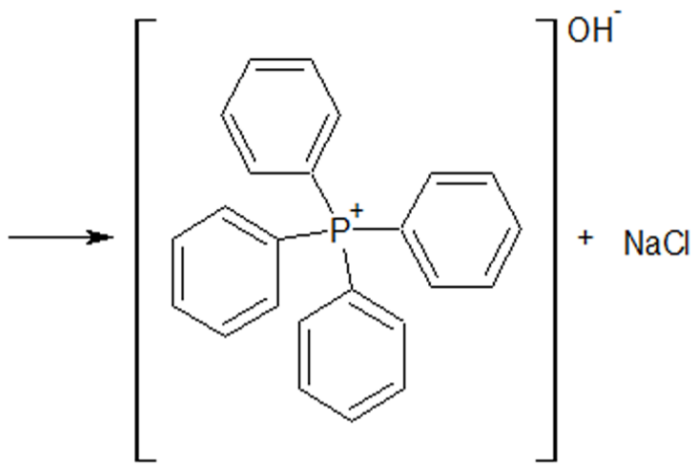

(BTPP-OH)

Figure 2: Reaction proposed for substitution of the chlorate ion of the BTPPC by the hydroxide ion and the generating of the BTPP-OH.<smiles>COc1ccc(C(=O)c2ccc(OC)cc2)cc1</smiles>

(DHBP-deprotonated)<smiles>O=P(Cl)(Cl)c1ccccc1</smiles>

(PPDC)<smiles>CC(C)(C)C</smiles><smiles>COc1ccc(C(=O)c2ccc([O-])c(I)c2)cc1</smiles><smiles>CCCCCOP(=O)(Oc1ccc(C(=O)c2ccc(C)cc2)cc1)c1ccccc1</smiles><smiles>CC(C)C</smiles><smiles>O=C(c1ccc(O)cc1)c1ccc(OP(=O)(Cl)c2ccccc2)cc1</smiles>

(DHBP-Polyphosfonate)

Figure 3: Mechanism proposed for the polymerization of the DHBP-Polyphosponate. 
Citation: Dominguini L, Martinello K, Peterson M, et al. (2019) Synthesis of Polyphosphate Polymer Employing the Bisphenol (BHBF) and the Dichloride of Phenylphosphonic (PPDC): Evaluation of the Thermal Characteristics. Current Trends Anal Bioanal Chem 3(1):114-124

Global reaction proposed is constituted by a series of chemical reactions. In first step occurs the anionic substitution of the chlorate ions in the BTPPC by the hydroxides ions originated of the dissociation of the $\mathrm{NaOH}$ compound, according Figure 2. For that reactions of anionic substitutions occur is necessary that the organic and aqueous phases are miscible. The ions of Benzyltriphenyl-phosphonium chloride are soluble in the organic solvents and not offer restrictions for the anionic substitutions and the BTPP-OH produced during reactions contains the hydroxide ion and is the responsible by the change of the electrical charges across the interface between the organic and inorganic phases [12].

In the second step of the global synthesis the DHBP is solubilized in DCM is added to BTPP-OH. When the ion BTPP-OH is transferred to the organic phase the hydroxide ion keeps the neutrality of the solution. So, the hydroxide ion is transferred from the aqueous phases to the organic phase, promoting the reaction of deprotonating of the DHBP, according reaction proposed in the Figure 3.

In the sequences the PPDC is added in the reaction medium containing DHBP-deprotonated. The PPDC have the $\mathrm{P}$ $=0$ chemical bond and characterizes the phosphorus as an electropositive agent and can interact easy with a nucleophilic center. The group $\mathrm{P}=\mathrm{O}$ have two leaving groups of $\mathrm{Cl}^{-}$, that allows a nucleophilic substitution by reaction SN2. So, the ion produced in the protonation process of the DHBP acts as a nucleophilic center and reacts with the PPDC to originate the DHBP-Polyphosphonate. So, a polyphenyl phosphonate is formed by successively reactions of the DHBP deprotonated with the two actives positive center of the PPDC.

A sequence of results proves the formation of a polyphenyl phosphonate polymer proposed by this work. The Figure 4 shows the FTIR spectrumsin range 500 to $1750 \mathrm{~cm}^{-1}$ obtained for the samples of polyphenyl phosphonate polymer and in the Figure 5 with the range 2000 to $4000 \mathrm{~cm}^{-1}$.

FTIR Spectrums shows transmittance bands typical of a polyphenyl phosphonated compound. These results are strong evidences of the occurrence of the synthesis of a polyphenyl phosphonate polymer, according proposed by global synthesis reaction. But, characteristic bands are observed too due the presence of the residual monomers and of the reagents.

In details it's possible to observe the presence of the bands in $1157 \mathrm{~cm}^{-1}$, related to the vibrational mode of the bonds type P-O- $\mathrm{C}_{\text {aromatic }}$ and the band in $989 \mathrm{~cm}^{-1}$ characteristics of the bonds type $\mathrm{O}=\mathrm{P}-\mathrm{C}_{\text {aromatic }}[13]$. The absence of the peaks in $601 \mathrm{~cm}^{-1}$ and in $524 \mathrm{~cm}^{-1}$ is a strongly evidence of the absence of the $\mathrm{P}-\mathrm{Cl}$ bonds in molecular structure and the low intensity peak in $3410 \mathrm{~cm}^{-1}$ is related the presence of the hydroxyls of the bisphenol [14].

FTIR band in $1436 \mathrm{~cm}^{-1}$ confirms the presence of bonds type $\mathrm{P}-\mathrm{C}_{\text {aromatic }}$ in the structure of polyphenyl phosphonate [15]. The $P=O$ bonds in the polymer are confirmed by the bands in $1315 \mathrm{~cm}^{-1}$ and $1273 \mathrm{~cm}^{-1}[12,13,16]$. The bands in 989 $\mathrm{cm}^{-1}$ is related to bonds type $\mathrm{O}=\mathrm{P}-\mathrm{C}_{\text {aromatic }}$ of the polyphenyl phosphonate [13].

Presence of others FTIR bands can be associated with others vibrational modes existents in the polyphenyl phosphonate or with the residual molecules present due the incomplete synthesis reactions. For example, the FTIR bands in 1597, 1504, 1116 e $719 \mathrm{~cm}^{-1}$ are associated with $\mathrm{C}=\mathrm{C}_{\text {aromatic }}$ bonds and the bands in $2918 \mathrm{~cm}^{-1}$ and in $848 \mathrm{~cm}^{-1}$ with the $\mathrm{H}$-Caromatic, while the bands in $1647 \mathrm{~cm}^{-1}$ are associated with the $\mathrm{C}=\mathrm{O}$ bonds, existents in the bisphenol [17].

Figure 6 shows details of the FTIR spectrum in the range of 1500 to $500 \mathrm{~cm}^{-1}$ obtained from the monomer

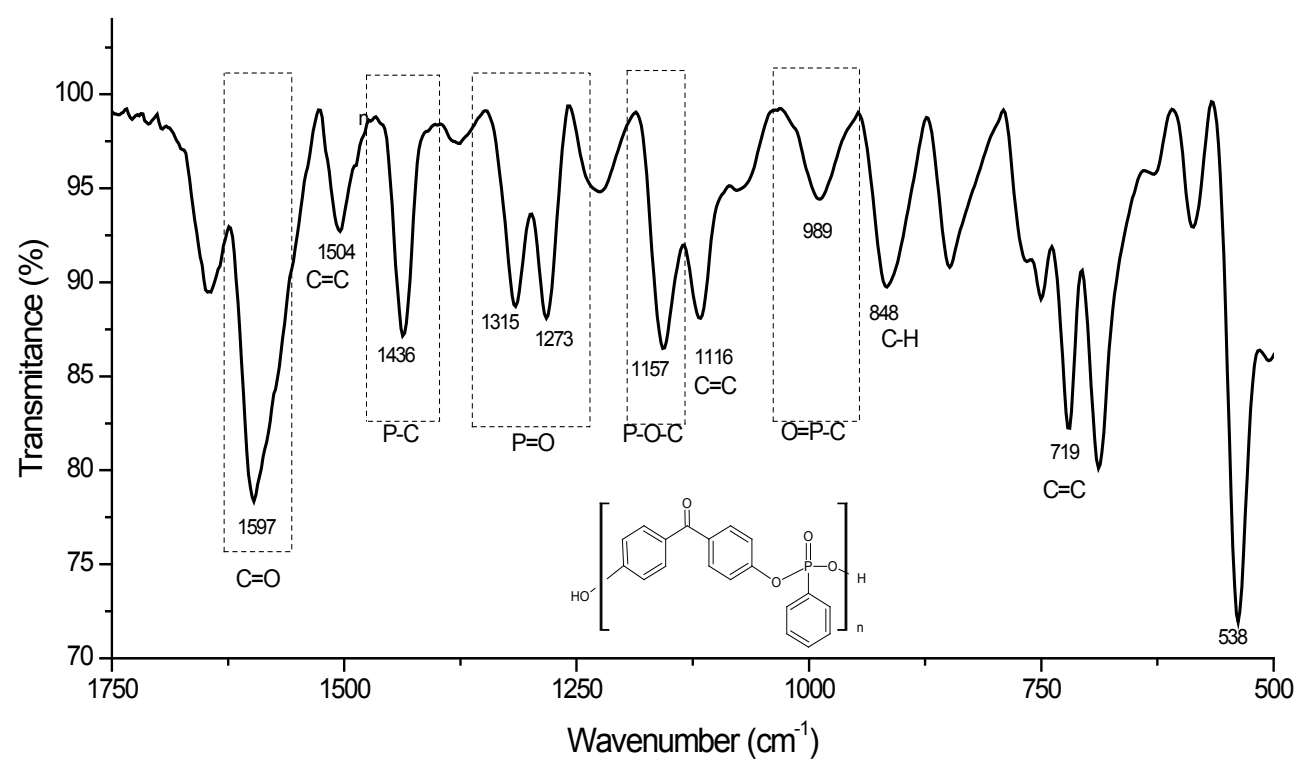

Figure 4: Details of the FTIR spectrum with the range 500 to $1750 \mathrm{~cm}^{-1}$ for the DHBP-Polyphosponate molecules. 


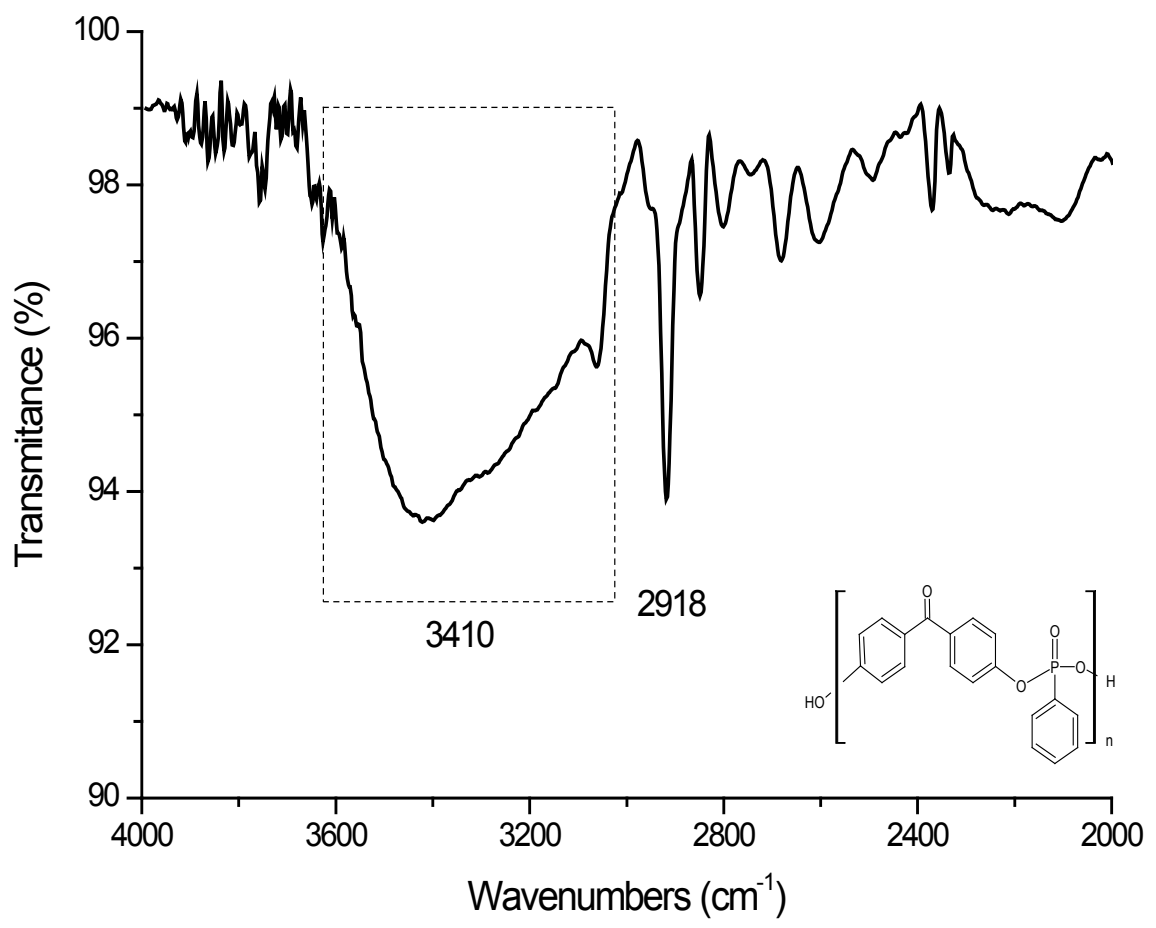

Figure 5: Details of the FTIR spectrum with the range 2000 to $4000 \mathrm{~cm}^{-1}$ for the DHBP-Polyphosphonated molecules.

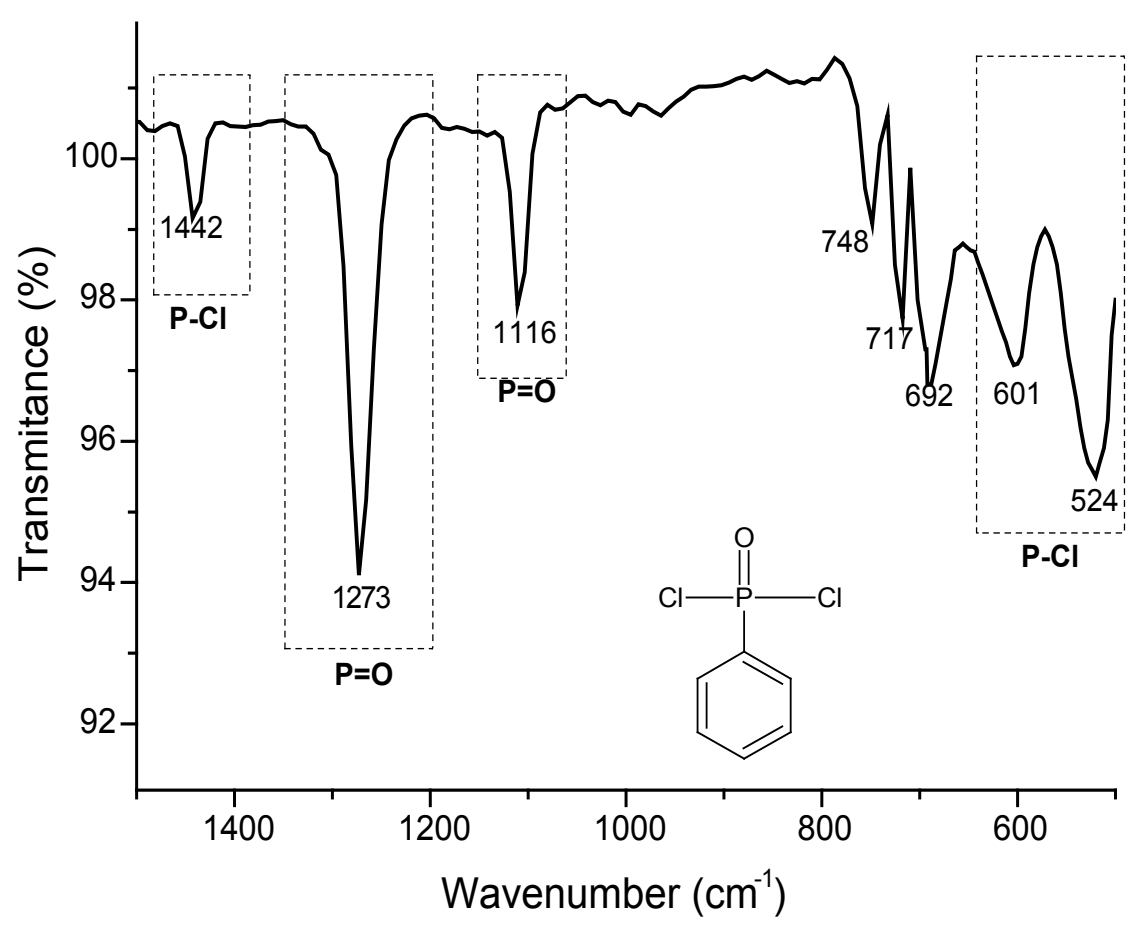

Figure 6: Details of the FTIR spectrum in the range of 1500 to $500 \mathrm{~cm}^{-1}$ obtained from the monomer Phenylphosphonic dichloride.

Phenylphosphonic Dichloride. It's possible to observe vibrational modes related to bonds of $\mathrm{P}-\mathrm{Cl}$ in $601 \mathrm{~cm}^{-1}$ and $524 \mathrm{~cm}^{-1}$. These transmittance bands not exists in the FTIR spectrum obtained for the polyphenyl phosphonate polymer and indicate that the bonds of $\mathrm{P}-\mathrm{Cl}$ were substituted by the bonds type $\mathrm{P}-\mathrm{O}$. Presence of the bonds $\mathrm{P}=\mathrm{O}$ in the monomer is confirmed by the presence of the bands in $1315 \mathrm{~cm}^{-1}$ and

\section{$1273 \mathrm{~cm}^{-1}[12,13,16]$.}

Figure 7 shows results of RMN 31P (phosphorus 31) obtained for the samples of polyphenyl phosphonate. The peak in $12.6 \mathrm{ppm}$ is related to the phosphorus elements bonded in the polymer chain and the peak in $12.1 \mathrm{ppm}$ refers to the phosphorus elements existents in the ends of the polymer 


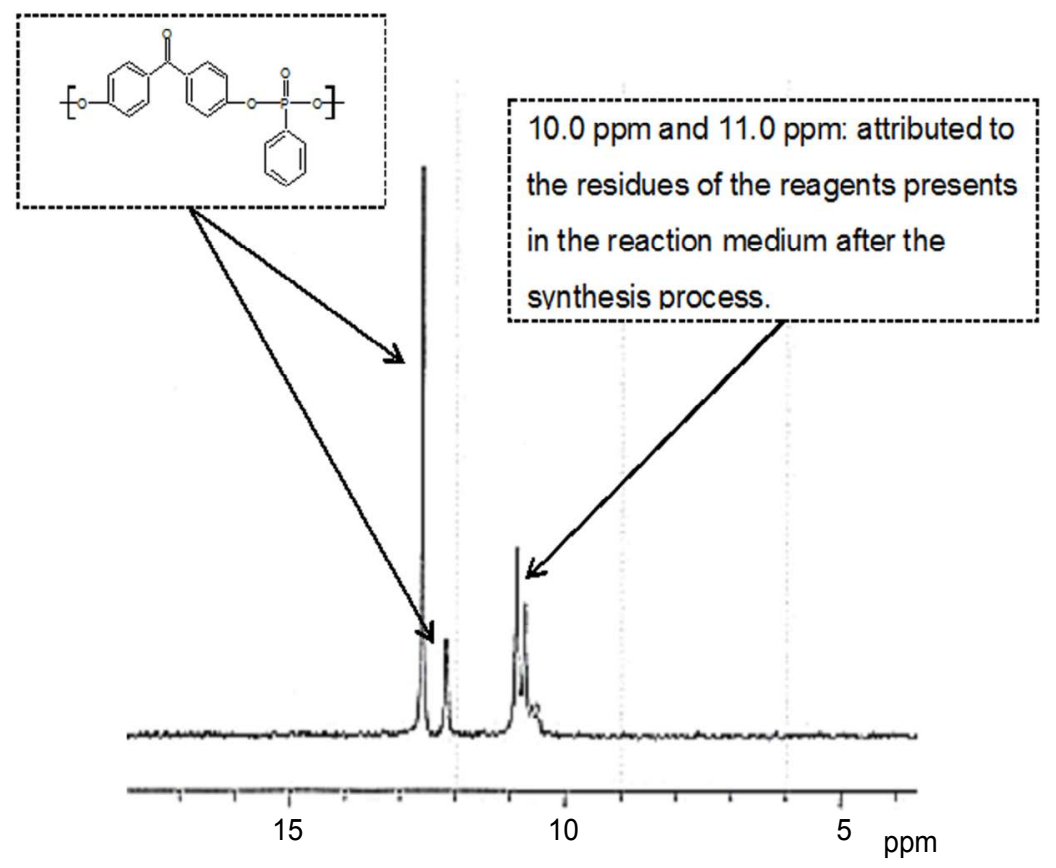

Figure 7: Spectrum of RMN 31P for the DHBP-Polyphosponate.

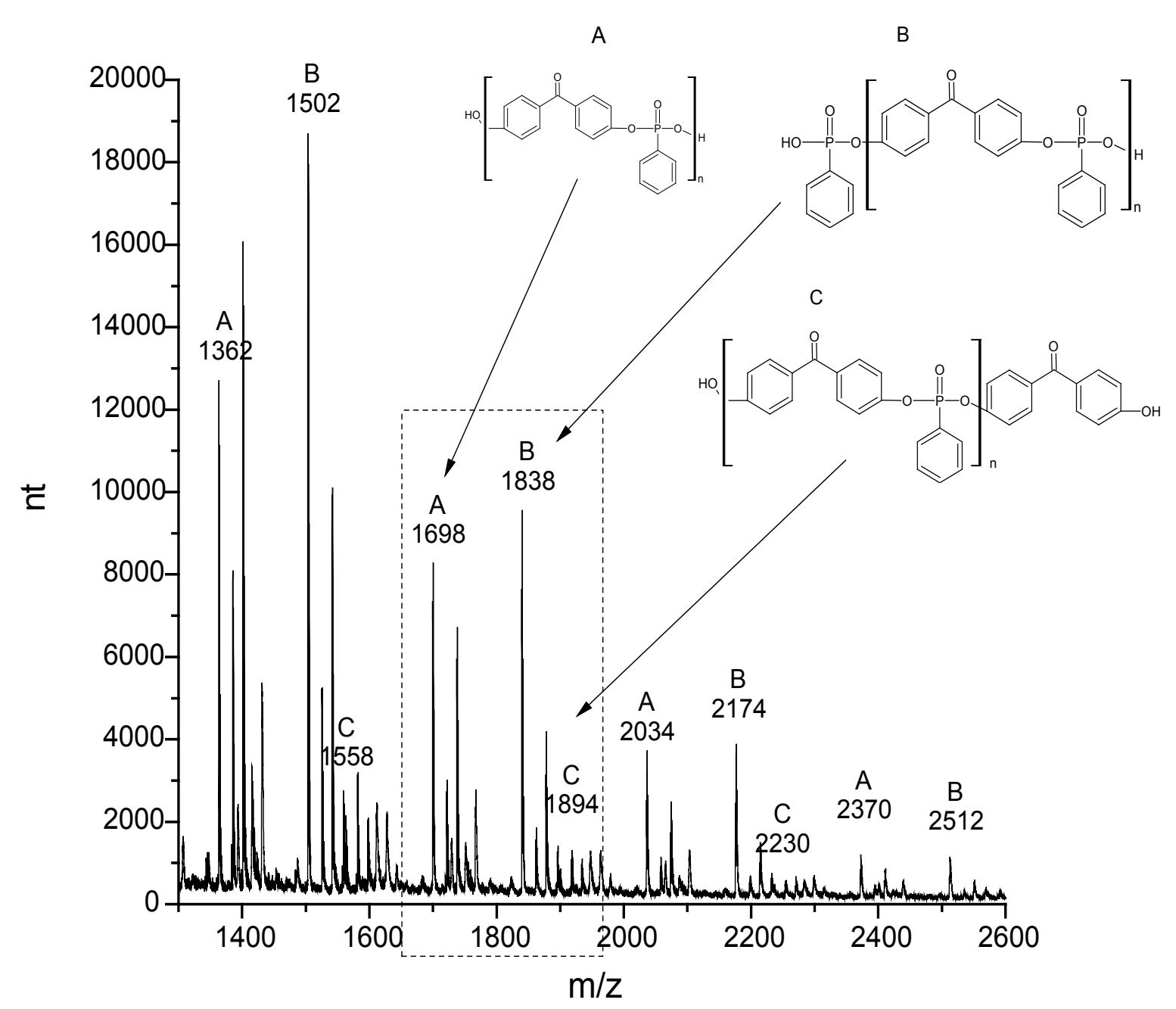

Figure 8: MALDI spectrum for the DHBP-Polyphosponate polymer. 
Citation: Dominguini L, Martinello K, Peterson M, et al. (2019) Synthesis of Polyphosphate Polymer Employing the Bisphenol (BHBF) and the Dichloride of Phenylphosphonic (PPDC): Evaluation of the Thermal Characteristics. Current Trends Anal Bioanal Chem 3(1):114-124

chain $[5,18]$. The endings of the polyphenyl phosphonate chain presents $\mathrm{P}-\mathrm{Cl}$ or $\mathrm{P}-\mathrm{OH}$ groups, being the chlorine and the oxygen the electron receptors. The extreme group of the polymer chain presents a RMN peak with smaller magnitude than the phosphonated group of the chain of the polymer [19]. Peaks between 10.0 and $11.0 \mathrm{ppm}$ can be attributed to the residues of the reagents presents in the reaction medium after the synthesis process. These evidences are showed too by FTIR results.

So, the RMN analys is associated with the FTIR results confirms the synthesis of a polyphenyl phosphonated polymer from the PPDC molecules.

Figure 8 shows the MALDI spectrum for the polymer synthesized. The result proves the formation of a DHBPPolyphosphonate polymer with molecular weight above 2000 atomic mass unit. The spectrum shows patterns for the repetitive units consistent for a polyphenyl phosphonate polymer containing its functional groups of finalization.

Pattern type $A$ is a set of peaks relative to a representative molecular unit present in the structure of the synthesized polymer. The MALDI spectrum shows configurations for these molecules containing amounts above 2000 units of measurement.

Pattern type $B$ and $C$ are the sets of peaks relative to the bonds between fundamental units of the polymer with the phosphonated groups or with a bisphenol group (benzophenone), respectively. The difference between peaks of the pattern $A$ and $B$ is of $140 \mathrm{~m} / \mathrm{z}$ and between the peaks of the pattern $A$ and $C$ is of $196 \mathrm{~m} / \mathrm{z}$ and coincides with the molecular weight of these molecules. This information proves that a phosphonated group and a bisphenol groups can bond with the polyphenyl phosphonate molecule in the end of its chain.

Analysis of the MALDI spectrum suggest three possible configurations for the polymers synthesized: i) Polyphenyl phosphonate containing 4,4'-dihydroxy-benzophenone in the chain - Pattern A; ii) Polyphenyl phosphonate with terminations of phosphonated groups - Pattern B and iii) Polyphenyl phosphonate with terminations of benzophenone group Pattern C. Figure 9 shows these possible molecular formulas.

MALDI results prove the occurrence of the polymerization reactions with the formation of polymeric molecules containing phosphorated structures, in accordance with FTIR and NMR analysis.

\section{A}<smiles>CC(C)c1ccc(C(=O)c2ccc(OC(=O)c3ccccc3)cc2)cc1</smiles>

:DHBP-polyphenylphosfonate - Pattern A

B<smiles>COP(=O)(O)c1ccc(C(=O)c2ccc(OP(=O)(OC)c3ccccc3)cc2)cc1</smiles>

: DHBP-polyphenylphosfonate with phosphorated groups as termination on the polymeric molecules - Pattern $B$<smiles>Cc1ccc(C(=O)c2ccc(OP(=O)(Oc3ccc(C(=O)c4ccc(O)cc4)cc3)c3cccc(C(C)O)c3)cc2)cc1</smiles>

: DHBP-polyphenylphosfonate with bysphenol groups as termination on the polymeric molecules - Pattern C

Figure 9: Possible configurations for the three types of the polyphenyl Phosphonate polymeric molecules. 


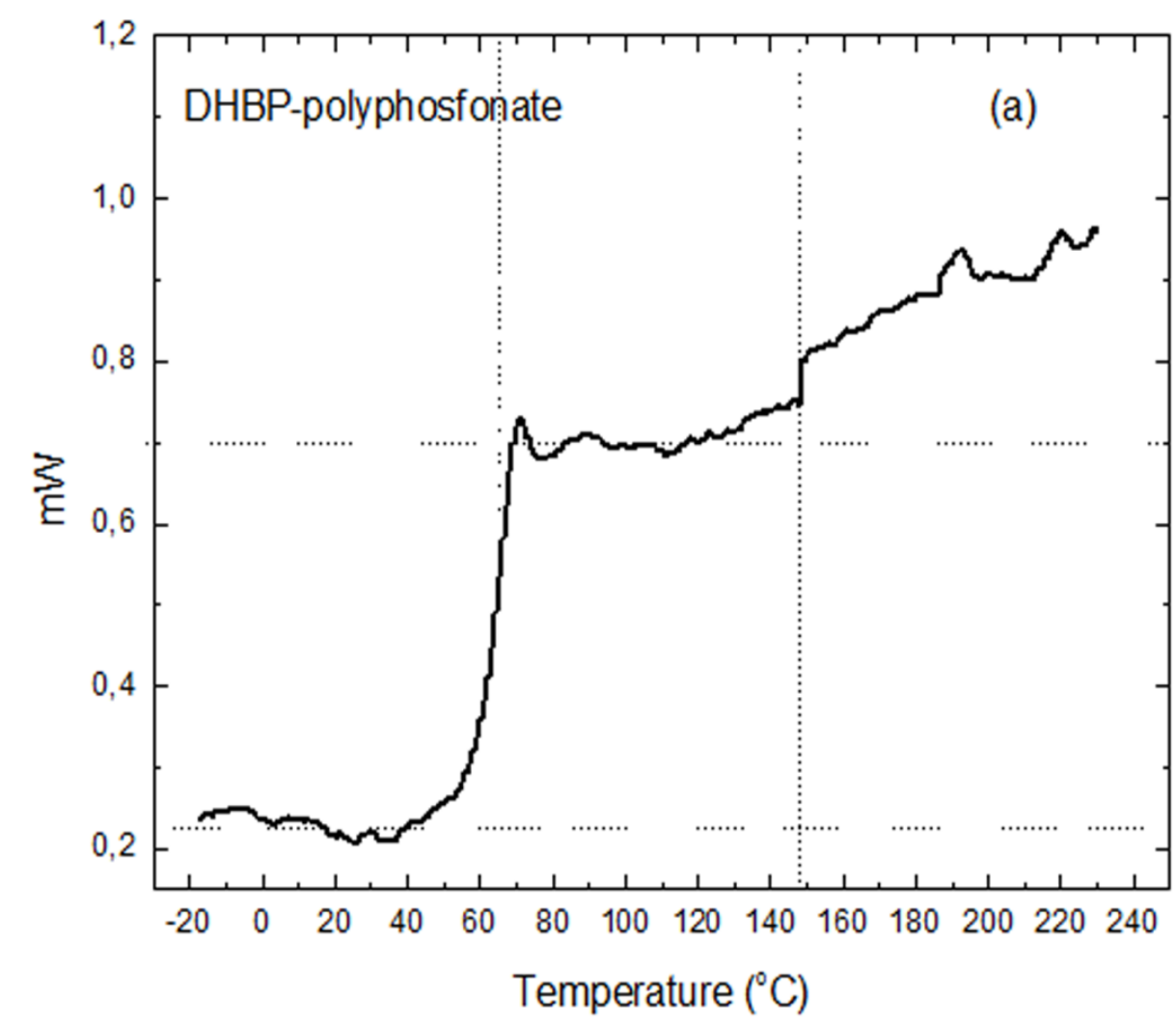

Figure 10a: Thermogram obtained by the Thermal Differential Analysis (DSC) for the DHBP-Polyphosponate polymer.

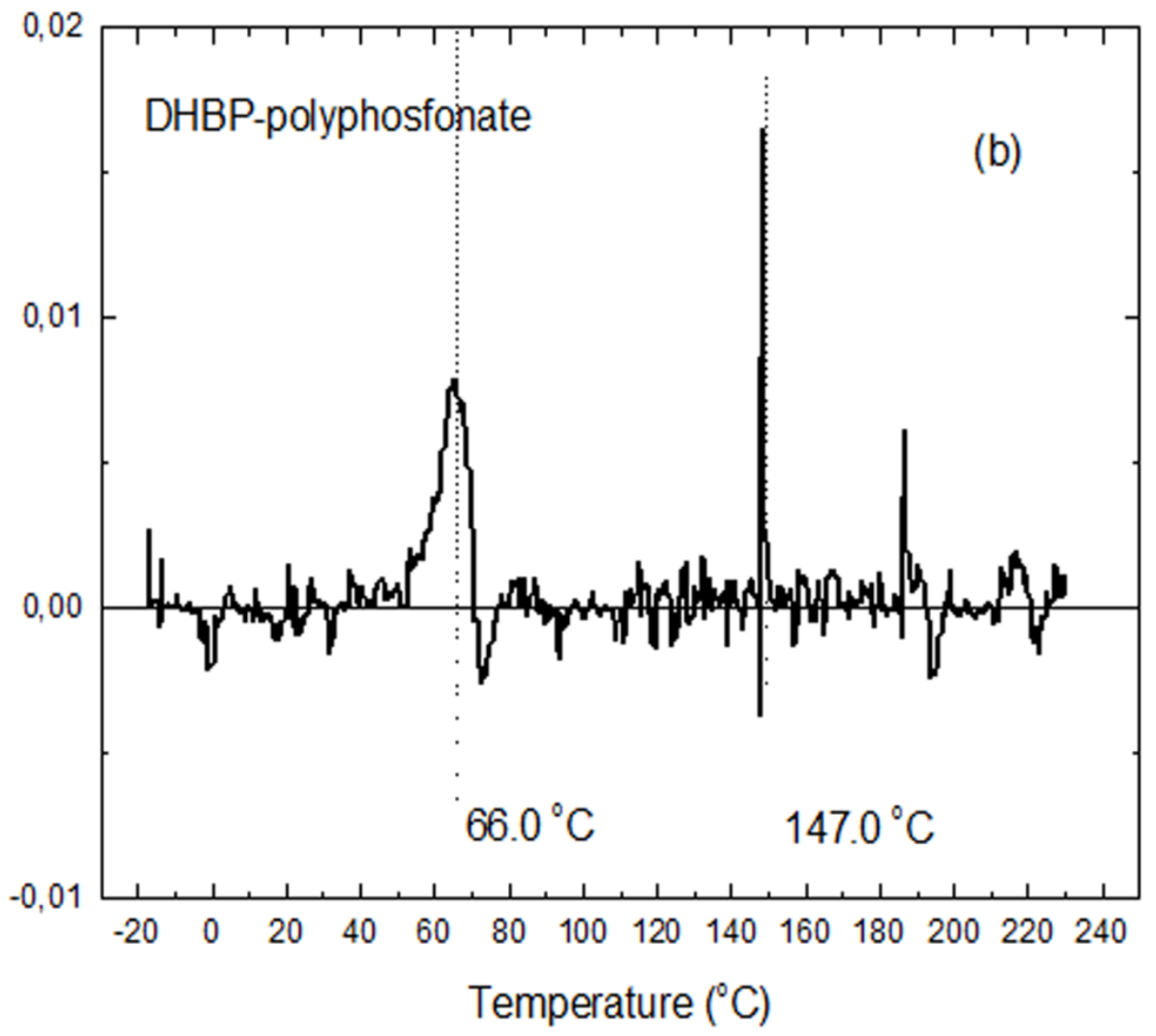

Figure 10b: Respective first order differential diagram for the signal of the DSC thermogram. 
Citation: Dominguini L, Martinello K, Peterson M, et al. (2019) Synthesis of Polyphosphate Polymer Employing the Bisphenol (BHBF) and the Dichloride of Phenylphosphonic (PPDC): Evaluation of the Thermal Characteristics. Current Trends Anal Bioanal Chem 3(1):114-124

Polyphenyl phosphonate molecules are formed by aromatic functional groups and is expected that its crystalline structures are amorphous. The Figure 10a shows the DSC thermograms obtained for a polymeric sample synthesized and the Figure $10 \mathrm{~b}$ its respectively first order differential diagram. It's possible to observe only vitreous transitions for the polyphenyl phosphonate molecules that occur between at temperatures $60.0^{\circ} \mathrm{C}$ to $70.0{ }^{\circ} \mathrm{C}$. For temperatures above $140.0^{\circ} \mathrm{C}$ occurs the thermal decomposition of the molecules with lower molecular weight and of the residual compounds due incomplete synthesis reactions.

DSC thermogram is typical of an amorphous compound and not shows signals of phase transformations. The first order differential diagram shows that the average temperature associated with the vitreous transitions of the polyphenyl phosphonate polymer is $66.0^{\circ} \mathrm{C}$ and above of $147.0^{\circ} \mathrm{C}$ the reactions of thermal degradations are accentuated.

Figure 11 shows the Thermal analyses (TGA) for the polyphenyl phosphonate. The results indicate the mass loss in function of the temperature. The mass loss at temperature of $147.0{ }^{\circ} \mathrm{C}$ is due the decomposition of the residual compounds from the incomplete synthesis reaction and confirms the results of the Differential Thermal Analysis. The greatest mass loss is observed above the temperature $220.0^{\circ} \mathrm{C}$ that indicates the start of the thermal decomposition of the phosphorated polymer.

Thermograms show too the presence of a significant quantities of residual mass after thermal decomposition of the phosphorated polymer. After heating until $700.0{ }^{\circ} \mathrm{C}$ the residual mass is approximately of $24.0 \mathrm{wt} \%$. This characteristic is very important if the future application of this polymer is as flame retardant additive in polymeric matrices. In these applications the residues after thermal decomposition can favor an intumescent effect in the polymeric matrix and generate a physical barrier for the oxygen into the polymeric matrices. This process inhibits with good efficiency the combustions processes of the polymers.

Phosphorated compounds are applied as flame retardant by having phosphorus elements in its structure [7]. The phosphorus elements favor the formation of an inhibitors layer on the surface of the polymer matrices during the combustions processes. These mechanisms decreases the heat generation during the combustion and reduces the contact area between polymeric molecules and the oxidizing agents, particularly with the oxygen. The predominant mechanisms in this processes depends of the characteristics of the polymer matrix and of the structure of the phosphorated compound applied [20].

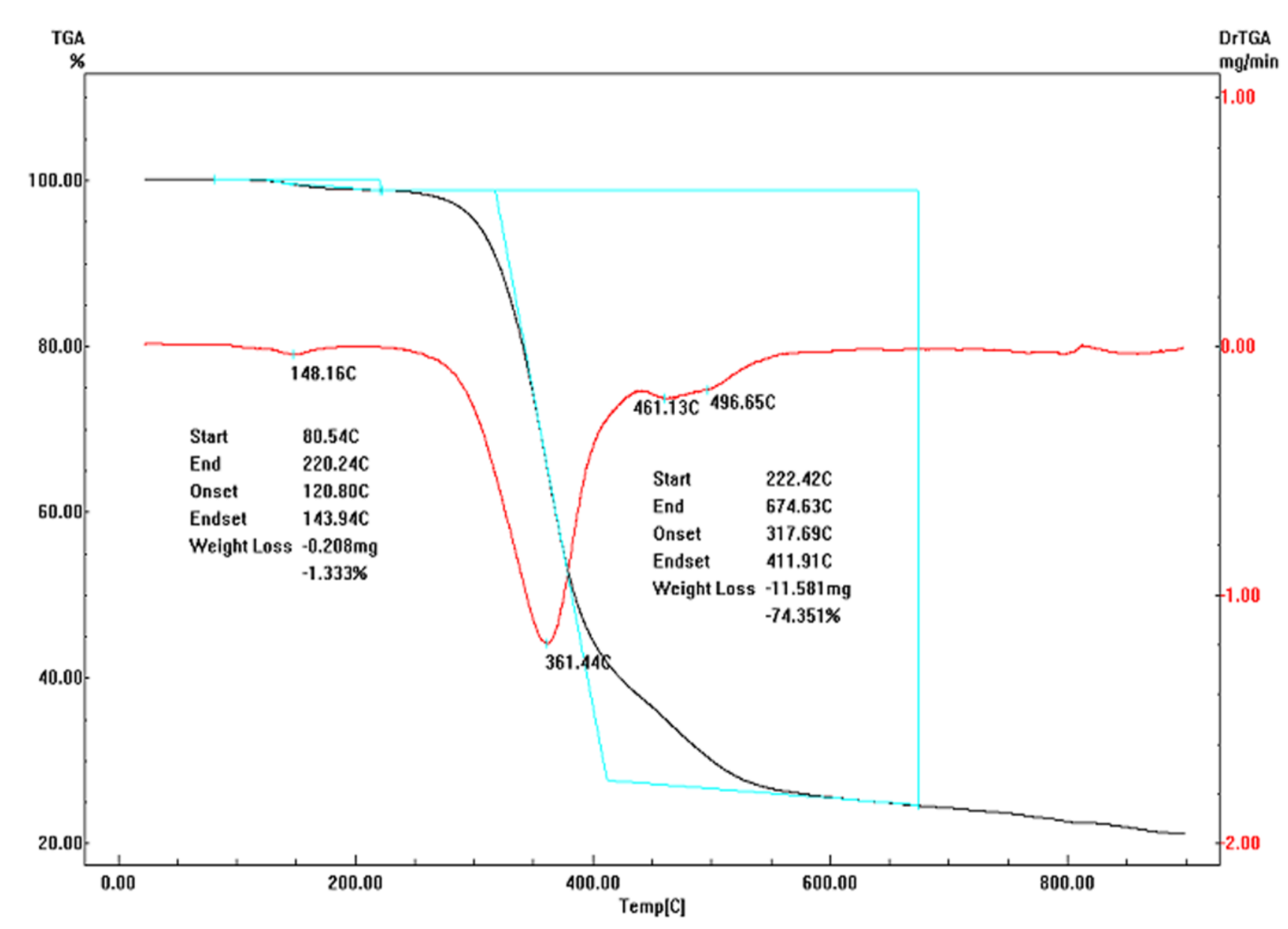

Figure 11: Thermogram obtained by the Thermogravimetry Analysis (TGA) for the polyphenyl Phosphonate in nitrogen atmosphere and heating rate of $10.0^{\circ} \mathrm{C} / \mathrm{min}$. 
Citation: Dominguini L, Martinello K, Peterson M, et al. (2019) Synthesis of Polyphosphate Polymer Employing the Bisphenol (BHBF) and the Dichloride of Phenylphosphonic (PPDC): Evaluation of the Thermal Characteristics. Current Trends Anal Bioanal Chem 3(1):114-124

Table 2: Relationship of phosphorus quantities in the structure of the DHBP-Polyphosphonate and in others phosphorated compounds and the amount of residual mass generated after their thermal decomposition.

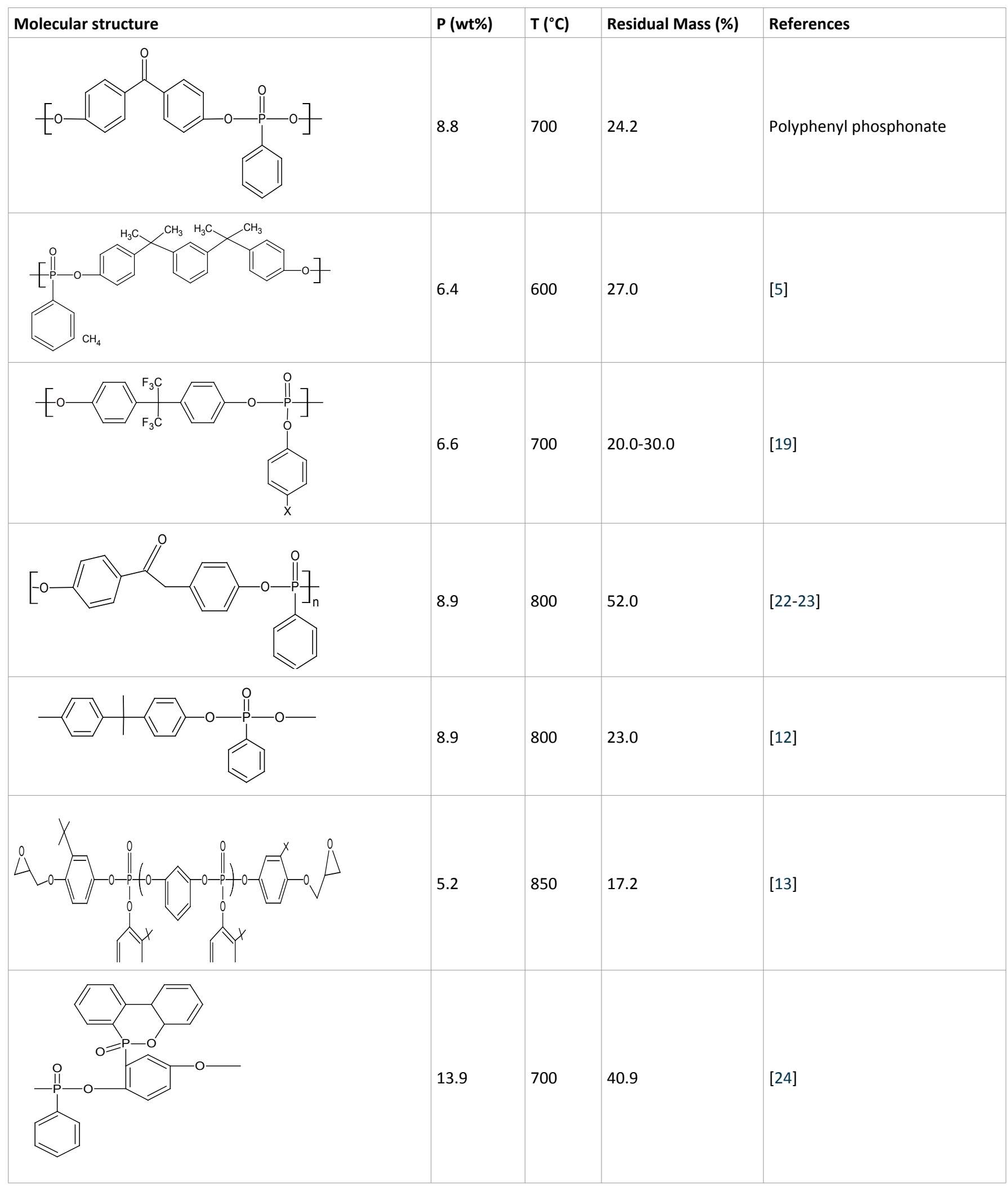

In general, the mass of the residues generated after the thermal decomposition of the polyphenyl phosphonate compounds are directly related by the amounts of phosphorus elements in the polymeric chain [21]. During the combustions reactions occurs the formation of a carbonaceous structure, that is an inhibitors layer (solid chair) originated by the pyrol- ysis reaction. The decomposition of the polyphosphate polymer releases radicals of $\mathrm{PO} \bullet, \mathrm{P} \bullet$ and $\mathrm{P}_{2}$ that have the ability of capturer radicals of $\mathrm{H} \bullet, \bullet \mathrm{O} \bullet$ and $\mathrm{HO} \bullet$. These phenomena allow the formation of high amounts of the residual mass after the thermal decomposition, at high temperatures even in inert atmosphere [22]. 
Citation: Dominguini L, Martinello K, Peterson M, et al. (2019) Synthesis of Polyphosphate Polymer Employing the Bisphenol (BHBF) and the Dichloride of Phenylphosphonic (PPDC): Evaluation of the Thermal Characteristics. Current Trends Anal Bioanal Chem 3(1):114-124

Mass loss is significant after thermal decomposition of the polymer synthesized and the quantities is similar to others flame retardants applied in polymers. The Table 2 relate the results obtained to polyphenyl phosphonate synthesized in this work with others phosphorated materials obtained by others scientific works $[23,24]$.

\section{Conclusions}

Results showed that by the interfacial polycondensation synthesis methods and utilizing a phenylphosphonic as monomer is possible to obtain a polymer containing phosphorus in its principal chain and with molecular weight higher than 2000 units. FTIR, RMN 31P and MALDI spectroscopies proved the formation of the DHBP-Polyphosponate polymer employing the Bisphenol (BHBF) and the Dichlorate of phenylphosphonic (PPDC).

Thermal analysis showed that the DHBP-Polyphosponate has good thermal stability until temperatures of $220.0^{\circ} \mathrm{C}$ and after its thermal degradation the residual mass is approximately of $24.0 \%$. These results potentiate the application of the DHBP-Polyphosponate for the future as good flame retardant additive for the polymer materials.

\section{Acknowledgements}

The authors thanks the Laboratório de Materiais Avançados e de Processos (LMPP-UNESC) and the Laboratório de Materiais e Corrosão (LaMAC - UFSC). Also thank the Fundo de ApoioàManutenção e aoDesenvolvimento da Educação Superior - FUMDES by financial incentive and the National Council for Scientific and Technological Development -CNPq for the financial support of the work.

\section{References}

1. Lu S Hamerton I (2002) Recent developments in the chemistry of halogen-free flame retardant polymers. Prog Polym Sci 27: 1661-1712.

2. Hoang D, Kim J, Polym Degrad Stab (2002) Synthesis and applications of biscyclic phosphorus flame retardants. Polymer Degradation \& Stability.

3. Hoang D, Kim J, Jang BN (2002) Synthesis and performance of cyclic phosphorus-containing flame retardants. Polym Degrad Stab 93: 2042-2047.

4. Wang $Q$, Shi W (2006) Photopolymerization and thermal behaviors of acrylated benzenephosphonates/epoxy acrylate as flame retardant resins. Eur Polym J 42: 2261-2269.

5. Iliescu S, Plesu N, Popa A, et al. (2011) Green synthesis of polymers containing phosphorus in the main chain. Chimie 14 : 647-651.

6. Kim J, Lee K, Lee K, et al. (2003) Studies on the thermal stabilization enhancement of ABS; synergistic effect of triphenyl phosphate nanocomposite, epoxy resin, and silane coupling agent mixtures. Polym Degrad Stab 79: 201-207.

7. Laoutid Fouad, Bonnaud Leila, Alexandre Michael, et al. (2009) New prospects in flame retardant polymer materials: From fundamentals to nanocomposites. Mat Sci Eng 63: 100-125.
8. Van Der Veen I, De Boer J (2012) Phosphorus flame retardants: Properties, production, environmental occurrence, toxicity and analysis. Chemosphere 88: 1119-1153.

9. Hsiue GH, Liu YL, Tsiao J J (2000) Unsaturated polyester resins: Numerical solution of a system of equations able to describe both styrene and unsaturation consumption. Appl Polym Sci 78: 124-132.

10. Allinger NL (1971) Organic Chemistry. Rio de Janeiro.

11. Wang L-S, Wang X-L, Yan G-L (2000) Synthesis, characterisation and flame retardance behaviour of poly(ethylene terephthalate) copolymer containing triaryl phosphine oxide. Polymer Degradation and Stability. Polym Degrad Stab 69: 127-130.

12. Ranganathan T, Joseph Zilberman, Richard J Farris, et al. (2006) Synthesis and characterization of halogen-free antiflammable polyphosphonates containing 4,4'-Bishydroxydeoxybenzoin. Macromolecule 39: 5974-5975.

13. Jeng R-J, SH Shau, YC Julian Chiu, et al. (2002) Flame retardant epoxy polymers based on all phosphorus-containing components. Eur Polym J 38.

14. Tao K, Li J, Xu L, et al. (2011) A novel phosphazene cyclomatrix network polymer: Design, synthesis and application in flame retardant polylactide. Polym Degrad Stab 96: 1248-1254.

15. Semenzato $S$ (2009) Identifying sources of respirable quartz and silica dust in underground coal mines in southern West Virginia, western Virginia, and eastern Kentucky. Appl Clay Sci 44: 110-118.

16. Ranganathan T, Bon-Cheol Ku, Joseph Zilberman, et al. (2007) Poly(arylate-phosphonate) copolymers with deoxybenzoin in the backbone: Synthesis, characterization, and thermal properties. J Polym Sci Chem 45: 4573-4580.

17. Ellzey KA, T Ranganathan, Joseph Zilberman, et al. (2006) Deoxybenzoin-Based Polyarylates as Halogen-Free Fire-Resistant Polymers. Macromolecules 39: 3553.

18. Liaw D-J, Chen P (1995) Synthesis of fluorine-containing polyphosphonates: low temperature solution polycondensation of bisphenol AF and phenylphosphonic dichloride. Polymer 36: 4491-4495.

19. Liaw D-J, Wang D-W (1996) Synthesis of fluorine-containing polyphosphates: low-temperature solution polycondensation of bisphenol AF and aryl phosphorodichloridates. Reac Funct Polym 30: 309-315.

20. Maiti S, Banerjee S, Palit SK (1993) Phosphorus-containing polymers. Progr Polym Sci 18: 227-261.

21. Wang H, Xu S, Shi W (2009) Photopolymerization behaviors of hyperbranched polyphosphonate acrylate and properties of the UV cured film. Prog Org Coat 65: 417-424.

22. Cheng L, Wang Y-Z (2010) Aryl Polyphosphonates: Useful HalogenFree Flame Retardants for Polymers. Materials 3: 4746-4760.

23. Ranganathan T, Michael Beaulieu, Joseph Zilberman (2008) Thermal degradation of deoxybenzoin polymers studied by pyrolysis-gas chromatography/mass spectrometry. Polym Degrad Stab 93: 1059-1066.

24. Wei, L-L, Wang, et al. (2011) Effect of a phosphorus-containing flame retardant on the thermal properties and ease of ignition of poly(lactic acid). Polym Degrad Stab 96: 1557-1561.

DOI: $10.36959 / 525 / 446$

Copyright: (C) 2019 Dominguini L, et al. This is an open-access article distributed under the terms of the Creative Commons Attribution License, which permits unrestricted use, distribution, and reproduction in any medium, provided the original author and source are credited. 